\title{
Comparative assessment of gene-specific variant distribution in prenatal and postnatal cohorts tested for Noonan syndrome and related conditions
}

\author{
N. T. Leach, PhD ${ }^{1}$, D. R. Wilson Mathews, MS, LCGC ${ }^{1}$, L. S. Rosenblum, PhD ${ }^{1}$, Z. Zhou, PhD ${ }^{1}$, \\ H. Zhu, PhD ${ }^{1}$ and R. A. Heim, PhD ${ }^{1}$
}

Purpose: To compare the pattern of gene-specific involvement and the spectrum of variants observed in prenatal and postnatal (mean $\pm \mathrm{SD}, 8.9 \pm 9.4$ years) cohorts tested for Noonan syndrome and related conditions.

Methods: Outcomes of sequencing panel testing were compared between prenatal $(n=845)$ and postnatal $(n=409)$ cohorts.

Results: PTPN11 and SOS1 harbored the majority of observed variants in both prenatal and postnatal cohorts, and $B R A F, H R A S$, KRAS, MAP2K1, MAP2K2, RAF1, and SHOC2 had similarities in their pattern of involvement in both cohorts. PTPN11 was the largest contributor of pathogenic variants and had the lowest frequency of variants of uncertain significance (VUS). SOS1 had the highest VUS frequency in both cohorts. The overall VUS frequency was twice as high in prenatal specimens (58.1 vs. 29.3\%). PTPN11 and SOS1 had a 1.5-fold higher VUS frequency in the prenatal cohort (10.7 vs. $7.4 \%$ and 95 vs. $61.1 \%$, respectively). The diagnostic yield was $3.7 \%$ for prenatal samples, with a higher yield of $12.3 \%$ in fetuses with cystic hygroma as a sole finding, and $21.3 \%$ for postnatal.

Conclusion: Comparison of prenatal versus postnatal specimens demonstrates that the pattern of specific gene involvement is similar, whereas the classification spectrum of observed variants differs considerably.

Genetics in Medicine (2019) 21:417-425; https://doi.org/10.1038/s41436018-0062-0

Keywords: Noonan syndrome; Genetic testing; Prenatal testing; Detection rate; Clinical indication

\section{INTRODUCTION}

Noonan syndrome is a relatively common Mendelian disorder with a panethnic prevalence of 1/1000-2500 (ref. 1). It belongs to a group of closely related conditions, including Noonan syndrome with multiple lentigines, Noonan syndrome-like disorder with loose anagen hair, Costello syndrome, and cardiofaciocutaneous syndrome. Patients with Noonan syndrome and related conditions (NSRCs) share features of short stature, distinct facial appearance, congenital heart defects, cryptorchidism, skin pigmentation anomalies, variable degrees of intellectual impairment, and risk of malignancies. ${ }^{2-4}$ Frequent prenatal anomalies associated with NSRCs include lymphatic dysplasia, most commonly increased nuchal translucency (NT) or cystic hygroma, polyhydramnios, and congenital heart defects. ${ }^{5,6}$ NSRCs overlap in their clinical presentation due to a common molecular etiology affecting the RAS/MAPK (rat sarcoma/ mitogen-activated protein kinase) signaling pathway, which also places them in the broader clinical category of RASopathies. The RAS/MAPK signaling cascade regulates a wide range of important developmental processes that involve cell proliferation, differentiation, migration, senescence, and apoptosis. Consequently, pathogenic variants (PV) altering the RAS/MAPK pathway have a pleiotropic effect and impact multiple systems in affected patients. ${ }^{7}$ Although similar, each NSRC has its own specific manifestations and risks, including coagulation defects and increased risk of myelomonocytic leukemia in Noonan syndrome, higher incidence of ECG anomalies and hypertrophic cardiomyopathy in Noonan syndrome with multiple lentigines, hair anomalies and higher proportion of septal cardiac defects in Noonan syndrome-like disorder with loose anagen hair, ectodermal anomalies and more severe intellectual impairment in cardiofaciocutaneous syndrome, and increased risk of rhabdomyosarcoma in Costello syndrome. $^{8}$

Typically, NSRCs have an autosomal dominant pattern of inheritance and are caused by gain-of-function PV that enhance RAS/MAPK signaling. ${ }^{8,9}$ Multiple genes in the RAS/ MAPK pathway have well-established roles in NSRC etiology, and there is a degree of genotype-phenotype correlation, where particular genes or different PV within the same gene are predominantly associated with a particular NSRC or contribute to a specific disease manifestation. ${ }^{8,10}$ The most commonly mutated gene is PTPN11, a protein-tyrosine phosphatase that accounts for about $50 \%$ of classic Noonan syndrome and $90 \%$ of Noonan syndrome with multiple

\footnotetext{
${ }^{1}$ Integrated Genetics, Laboratory Corporation of America Holdings, 3400 Computer Drive, Westborough, Massachusetts, USA. Correspondence: N. L. Leach (Natalia. Leach@integratedgenetics.com)
} 
lentigines. ${ }^{11,12}$ PTPN11 PV are associated with pulmonary stenosis, bleeding diathesis, growth hormone anomalies, and juvenile myelomonocytic leukemia. ${ }^{13,14}$ SOS1, a guanine nucleotide exchange factor, is the second most commonly mutated gene responsible for about $10 \%$ of Noonan syndrome, and SOS1 PV confer an increased risk of pulmonary stenosis, embryonal rhabdomyosarcoma, and ectodermal anomalies. ${ }^{15-17} \mathrm{PV}$ in $R A F 1$, a serine-threonine kinase, confer a markedly increased risk of hypertrophic cardiomyopathy in Noonan syndrome and Noonan syndrome with multiple lentigines. ${ }^{18} B R A F$, also a serine-threonine kinase, is mutated in a majority of patients with cardiofaciocutaneous syndrome. PV in $M A P 2 K 1$ and $M A P 2 K 2$, effectors of RAF proteins, account for another significant proportion of this condition. ${ }^{19,20} \mathrm{PV}$ in HRAS, a GTPase, are seen predominantly in Costello syndrome and are associated with increased risk of rhabdomyosarcoma, neuroblastoma, and transitional cell carcinoma. ${ }^{21-23} \mathrm{~A} \mathrm{PV}$ residing in exon 2 of SHOC2, a scaffold protein, is found in patients with Noonan syndrome with loose anagen hair. ${ }^{24} \mathrm{PV}$ in another GTPase, KRAS, are observed in Noonan, Costello, and cardiofaciocutaneous syndromes, and appear to convey a more severe cognitive impairment. ${ }^{25}$ NSRCs heterogeneity is continuing to expand as more causative genes are identified by exome and genome sequencing of patients for whom molecular defects eluded detection via standard diagnostic panels. ${ }^{25-27}$ These new approaches allowed for a more recent identification of rare variants in genes not included in this study, such as RIT1, NRAS, SOS2, RASA2, A2ML1, RRAS, and LZTR1. ${ }^{26,28-34}$

Due to similarities in clinical presentation among NSRCs, combined with marked variable expressivity as well as a phenotypic overlap with other nonrelated conditions, diagnosis of NSRC can be challenging. Molecular genetic testing has become an essential tool in the differential diagnosis of patients and in the assessment of fetuses with ultrasound anomalies suggestive of NSRC. Pinpointing a mutation to a particular gene also has importance in providing specific management guidance, such as addressing increased risk of specific malignancies and hypertrophic cardiomyopathy, or treating coagulation defects and growth hormone anomalies. Multiple NRSCs panels, varying in gene number and content, are currently offered by many diagnostic entities. As prenatal molecular testing moves toward a noninvasive approach using cell-free fetal DNA in maternal circulation, one can expect further increases in the amount of fetal NRSC diagnostic testing.

Here, we present a comparative, large-scale analysis of NSRCs molecular testing in prenatal and postnatal cohorts. Sequence variant analysis was performed using a multigenic panel including BRAF, HRAS, KRAS, MAP2K1, MAP2K2, $P T P N 11, R A F 1, S O S 1$, and SHOC2. Although this panel is not inclusive of all the currently known NSRC genes, it serves the main objective of this study, which is an assessment of differences and similarities in the pattern of gene-specific involvement and the spectrum of observed variants between prenatal and postnatal cohorts among the tested genes. Such a comparative analysis allowed an investigation into whether prenatal and postnatal ascertainment changes the relative contribution of variants in each tested gene to the pool of detected variants. We also examined the variant classification spectrum by further parsing prenatal specimens by indication for testing. Our study showed that the gene-specific pattern of involvement had similarities between the prenatal and postnatal cohorts; however, the spectrum of identified variants was different. It demonstrates that ascertainment, including prenatal or postnatal context, and prenatal clinical indication, affect the classification spectrum of identified variants, with a consequent effect on the diagnostic yield.

\section{MATERIALS AND METHODS}

\section{Patient samples}

A total of 1254 unique and consecutively submitted cases comprising 845 prenatal and 409 postnatal specimens were analyzed retrospectively. The average age for postnatal patients was $8.9 \pm 9.4$ years. All cases were referred by clinicians for molecular NSRCs testing due to either clinical features or ultrasound anomalies suggestive of NSRCs. DNA was isolated from whole blood using the Qiagen QIAmp 96 DNA Blood Kit (Qiagen, http://www.qiagen.com) and Chemagic DNA blood kit (PerkinElmer, http://www. chemagen.com). DNA was isolated from direct or cultured amniotic fluid or chorionic villi sampling using a modified salting-out method. Absence of significant maternal cell contamination was assessed for all prenatal cases by short tandem repeat (STR) analysis using the PowerPlex ${ }^{\circledR} 16 \mathrm{HS}$ System (Promega, https://www.promega.com). Karyotype and chromosome microarray analysis results were assessed when available.

\section{NSRC testing}

Next-generation sequencing was performed using the Agilent ${ }^{\circledR}$ SureSelect ${ }^{\circledR}$ XT enrichment method (Agilent Technologies, http://www.genomics.agilent.com) and multiplex, paired-end sequencing on the Illumina $^{\circledR}$ next-generation sequencing platform (Illumina, https://www.illumina.com). A custom designed RNA bait library was used to capture regions of interest, which included all coding and flanking intronic sequences of BRAF, HRAS, KRAS, MAP2K1, $M A P 2 K 2$, PTPN11, RAF1, and SOS1 genes, and exon 2 of the SHOC2 gene. GRCh37/hg19 build of the human genome was used as a reference sequence. Trimming, duplicate removal, and alignment of sequencing reads as well as variant calling were done using the CLCBio Genomics Workbench software (Qiagen, https://www.qiagenbioinformatics.com). Sanger sequencing was used to confirm variants identified by next-generation sequencing and for follow-up parental testing: genomic DNA was amplified at targeted regions of interest, sequenced bidirectionally, and analyzed using SeqScape software (ThermoFisher Scientific, https://www. thermofisher.com). 


\section{Variant classification}

Classification of identified variants was determined using current American College of Medical Genetics and Genomics-Association for Molecular Pathology (ACMG/ AMP) standards and guidelines for the interpretation of sequence variants in conjunction with an internally developed protocol. ${ }^{35}$ In brief, the strength of evidence supporting an association with disease was assessed using an algorithmic approach that included the following elements: frequency in the general population derived from public databases and publications; occurrence in affected individuals: de novo occurrence and cosegregation with a disease in family studies; predicted functional effect based on in silico assessment by Blosum50, SIFT, PolyPhen-2, Mutation Taster, SNPs\&GO, and Alamut ${ }^{\mathrm{Tm}}$; and the functional effect based on published studies in controlled experimental systems. Additional considerations included gene-specific and disease-specific properties, such as disease prevalence and penetrance, the expected molecular mechanism, the variant location in well-established functional domains or hot spots, or co-occurrence with a known pathogenic variant. Identification of a pathogenic or likely pathogenic variant was considered a positive result. Benign and likely benign variants were considered negative results and not included in this analysis. The category of variant of uncertain significance (VUS) was further subdivided into VUS-possibly pathogenic, VUS-uncertain, and VUSpossibly benign. The subdivision of VUS into three subcategories allowed for further granularity in the interpretation related to variants with a higher or a lower likelihood of pathogenicity. Identification of a VUS without a co-occurring pathogenic or likely pathogenic variant was considered an inconclusive result.

\section{Calculations}

Overall positive rate or diagnostic yield was calculated as the percentage of the cases with a pathogenic or likely pathogenic variant relative to the total number of cases.

Gene-specific positive rate was calculated as the percentage of pathogenic and likely pathogenic variants in a particular gene relative to the total number of these variants observed across all the tested genes.

Overall VUS yield was calculated as the percentage of cases with a VUS (including VUS-possibly pathogenic, VUSuncertain, and VUS-possibly benign) relative to the total number of cases.

Overall VUS frequency was calculated as the percentage of total number of VUS observed across all the tested genes relative to the total number of variants observed.

Gene-specific VUS frequency was calculated as the percentage of VUS relative to the total number of variants observed in a particular gene.

Gene-specific VUS contribution was calculated as the percentage of VUS observed in a particular gene relative to the total number of VUS observed across all the tested genes.

\section{RESULTS}

To assess the pattern of gene-specific involvement and the spectrum of variants observed in prenatal and postnatal cohorts, sequence variants identified in 1254 clinical specimens submitted to our laboratory were analyzed retrospectively. Variants were found in all nine genes sequenced, with varying distributions and classifications.

\section{Prenatal cohort}

Eight hundred forty-five prenatal samples were analyzed. A total of 74 variants were found in 72 prenatal cases $(8.5 \%)$ (Supplementary Tables S1 and S2). Two cases carried two variants. PTPN11 harbored $37.8 \%$ (28) of all variants observed, which was the highest among the genes tested (Fig. 1a). SOS1 contained 27\% (20) of the detected variants, and the remaining genes accounted for a much lower number of variants: $9.5 \%$ (7) for $M A P 2 K 2,8.1 \%$ (6) for RAF1, 6.8\% (5) for BRAF, $4.1 \%$ (3) for HRAS, $2.7 \%$ (2) for KRAS and SHOC2 each, and only $1.4 \%$ (1) for MAP2K1 (Fig. 1a).

Among variants detected in prenatal specimens, 31 pathogenic and likely pathogenic variants were found in five of the genes tested: PTPN11, RAF1, SOS1, BRAF, and HRAS. The majority of these variants were in PTPN11, accounting for $80.7 \%$ (25) (Fig. 2a, Supplementary Table S1). The other four genes had a significantly smaller number of pathogenic and likely pathogenic variants, with only three variants found in $R A F 1$ (9.7\%), and a single pathogenic variant identified in each of SOS1, BRAF1, and HRAS (Fig. 2a).

The overall VUS frequency was $58.1 \%$ (43/74) in the prenatal cohort. The gene-specific VUS frequency varied widely. PTPN11 had the lowest VUS frequency of $10.7 \%$ $(3 / 28)$, whereas SOS1 had the highest VUS frequency of $95.0 \%$ $(19 / 20)$ (Supplementary Table S1). Half of the variants in RAF1 (3/6) were VUS, and the majority of variants were in the VUS category for BRAF (4/5) and HRAS (2/3). Only VUS were found in the remaining genes: MAP2K2 (7/7), KRAS (2/ 2), SHOC2 (2/2), and MAP2K1 (1/1) (Supplementary Table S1).

Among 43 identified VUS, SOS1 was the main contributor with gene-specific VUS contribution of $44.2 \%$ (19), followed by $M A P 2 K 2$ with $16.3 \%$ (7) and BRAF with $9.3 \%$ (4) (Fig. 2a). PTPN11 and RAF1 each had gene-specific VUS contribution of $7 \%$ (3), and the rest of the genes were minor contributors: $4.7 \%$ (2) for each of HRAS, KRAS, and SHOC2, and 2.3\% (1) for $M A P 2 K 1$ (Fig. 2a).

The distribution of abnormal ultrasound findings for prenatal NSRC testing is depicted in Fig. 3a. By far, the most common clinical indications in the 845 prenatal cases were increased NT in 53.8\% (455) and cystic hygroma in $22.4 \%$ (189). NT was reported as the sole finding in 51.2\% (433) and cystic hygroma in $19.2 \%$ (162) of all cases. Other ultrasound anomalies were found in addition to NT in $2.6 \%$ (22) and in addition to cystic hygroma in $3.2 \%$ (27) of cases. Increased nuchal fold, a second trimester marker associated with NSRCs, was present in $7.5 \%$ (63) of all cases, either as a single finding in $5.1 \%$ (43), or in association with additional findings in $2.4 \%$ 

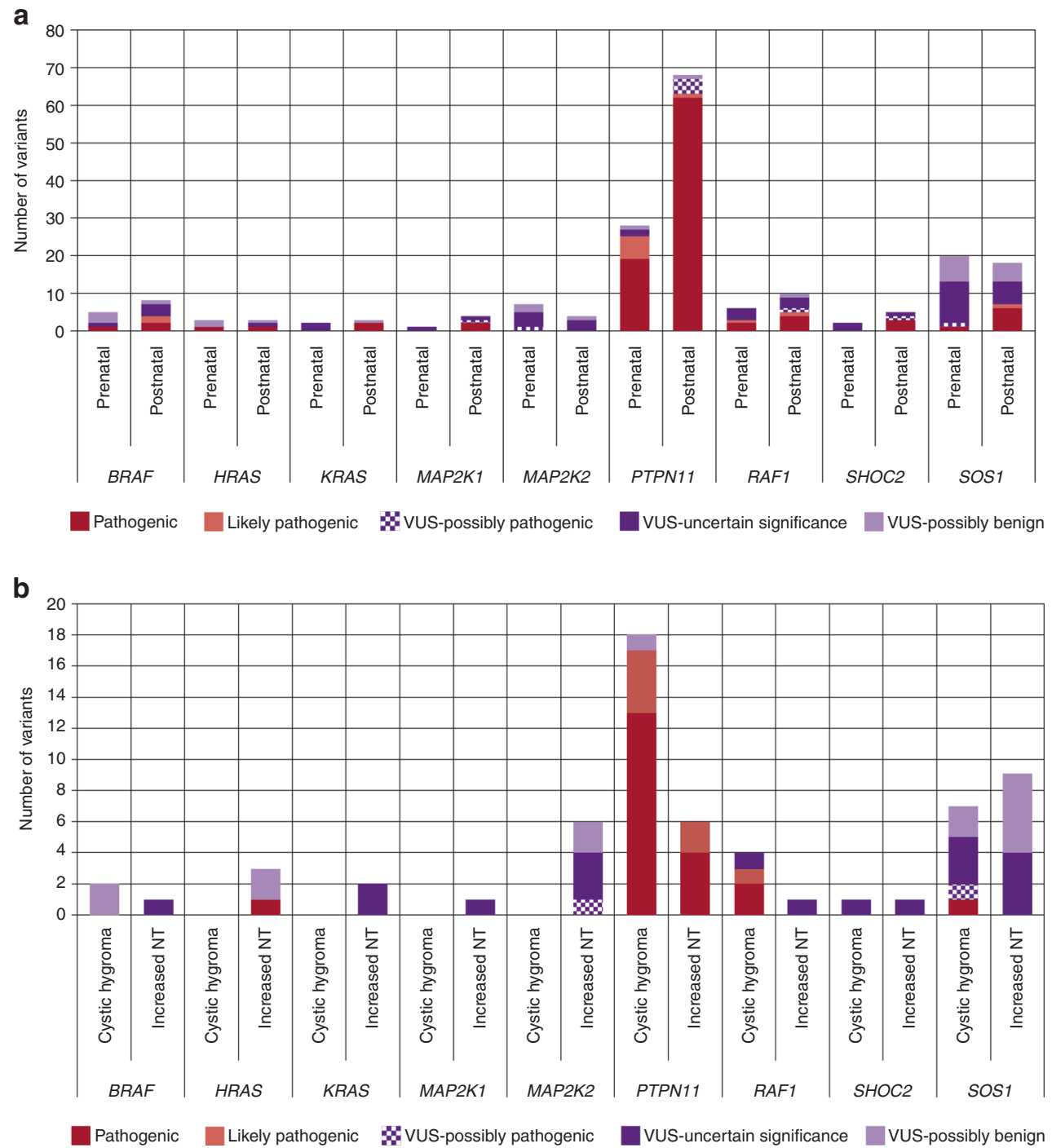

Fig. 1 Gene-specific variant distribution. a Gene-specific variant distribution in prenatal and postnatal specimens. b Gene-specific variant distribution in prenatal specimens with cystic hygroma and increased nuchal translucency (NT). VUS variant of uncertain significance

(20). Among other recurrent anomalies, heart defect was observed in $4.7 \%$ (40) of cases, either alone in $1.9 \%$ (16), or in combination with other findings in $2.8 \%$ (24). Lymphatic anomalies, such as edema, hydrops, pleural effusion, and polyhydramnios, were present in $2.4 \%$ (20) of fetuses. Among fetuses with positive results the predominant ultrasound findings were cystic hygroma and increased NT alone, which comprised $64.5 \%$ (20) and 20\% (6), respectively (Fig. 3b).

Because ultrasound markers associated with NSRCs are also found in chromosomal disorders, $84 \%$ (712) of prenatal cases had chromosomal studies performed by karyotyping and/or microarray analysis. These studies showed a normal result in $97 \%$ of cases (694). A single case was found to have an abnormal mosaic karyotype (48,XXY, +13[7]/46,XY[26]) as well as a pathogenic variant in PTPN11.

The overall positive rate or diagnostic yield for prenatal cases was $3.7 \%(31 / 845)$; however, if specimens are stratified by clinical indication, there are considerable differences. For the most common clinical indications, the highest positive rate was $12.3 \%(20 / 162)$ in fetuses with cystic hygroma as the sole ultrasound finding, and the lowest positive rate was $1.4 \%$ (6/ 433) in fetuses with increased NT as the sole ultrasound finding. Pathogenic and likely pathogenic variants were found in PTPN11, RAF1, and SOS1 in fetuses with cystic hygroma, and in PTPN11 and HRAS in fetuses with increased NT (Fig. 1b). The overall VUS yield for prenatal cases was 5.1\% (43/845). In two prenatal cases that carried more than one variant, a VUS co-occurred with a pathogenic variant. Therefore, inconclusive reports were issued for only $4.9 \%$ of prenatal cases.

\section{Postnatal cohort}

Four hundred nine postnatal samples were analyzed. A total of 123 variants were identified in 116 cases (28.4\%) (Supplementary Table S3). Seven cases carried two variants. Most of the variants were found in PTPN11 at 55.3\% (68), followed by SOS1 at $14.6 \%(18), R A F 1$ at $8.1 \%$ (10), and BRAF 
a

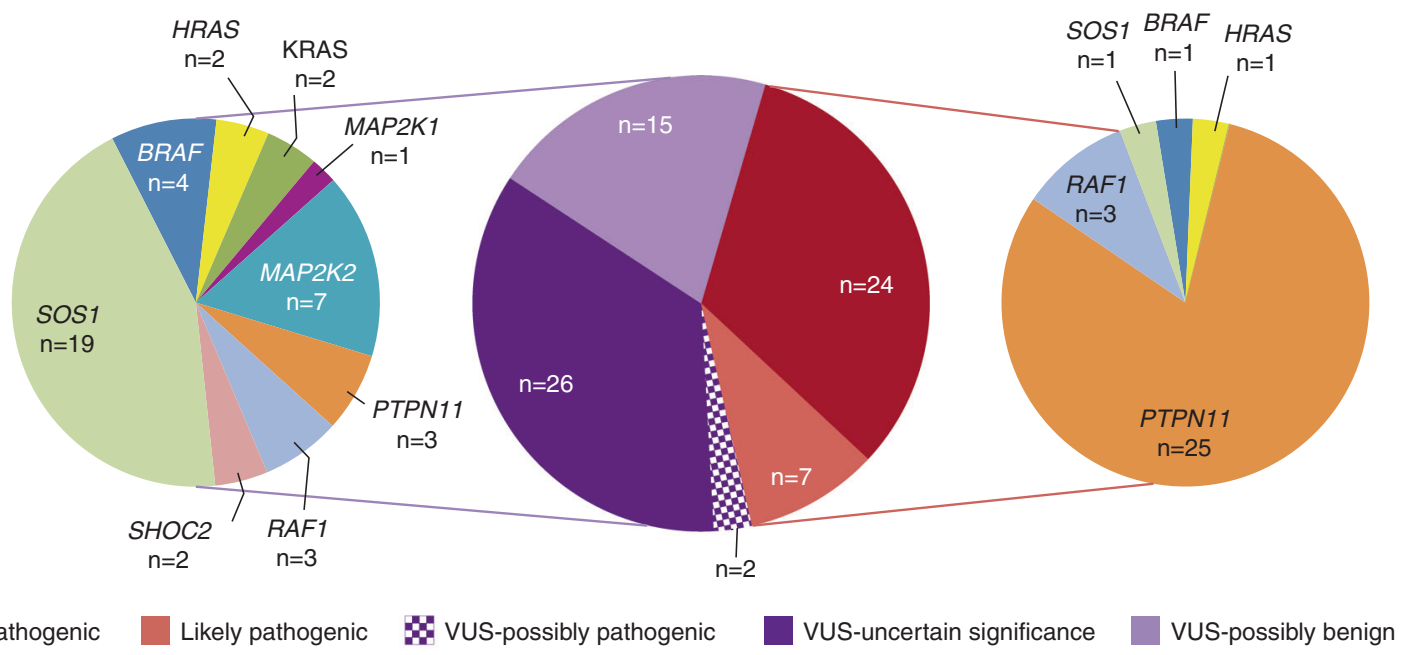

b

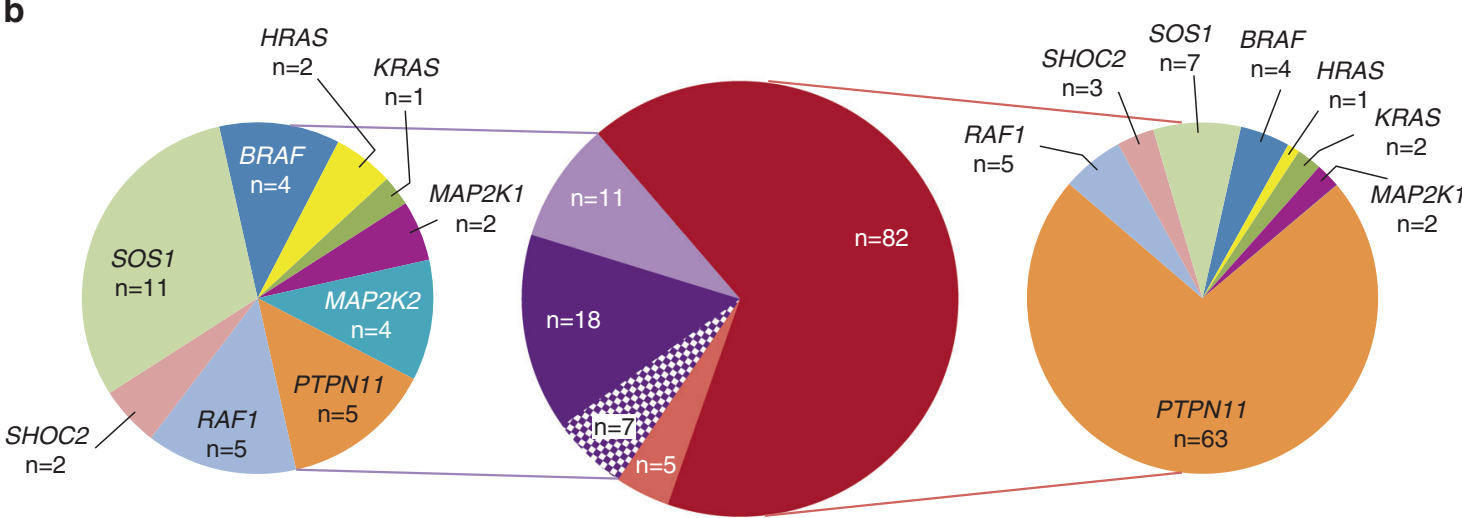

Pathogenic Likely pathogenic $\quad$ VUS-possibly pathogenic \ vUS-uncertain significance VUS-possibly benign

Fig. 2 Classification spectrum of detected variants. a Variants detected in prenatal specimens with their respective gene-specific contributions. b Variants detected in postnatal specimens with their respective gene-specific contributions. VUS variant of uncertain significance

at $6.5 \%$ (8) (Fig. 1a). The remaining genes had a considerably lower number of variants: SHOC2 at $4.1 \%(5), M A P 2 K 2$ and $M A P 2 K 1$ at $3.3 \%(4)$ each, and HRAS and KRAS at $2.4 \%$ (3) each (Fig. 1a).

Eighty-seven of the 123 variants $(70.7 \%)$ were pathogenic or likely pathogenic in postnatal samples and 63 of them were in PTPN11 (72.4\%) (Fig. 2b). The remaining 24 pathogenic and likely pathogenic variants were found in SOS1 (8.0\%), RAF1 (5.7\%), BRAF (4.6\%), SHOC2 (3.4\%), MAP2K1 (2.3\%), KRAS (2.3\%), and HRAS (1.1\%). No pathogenic or likely pathogenic variants were observed in MAP2K2 (Fig. 2b).

The overall VUS frequency across all genes in the postnatal cohort was $29.3 \%$ (36/123). The gene-specific VUS frequency was $61.1 \%(11 / 18)$ for SOS, and $50 \%$ for RAF1 and BRAF each (5/10 and 4/8, respectively) (Supplementary Table S1). PTPN11 had the lowest gene-specific VUS frequency with only $7.4 \%(5 / 68)$ of all variants identified in this gene being VUS (Supplementary Table S1). VUS frequencies were not evaluated for the remaining genes as the number of variants identified was too small to produce an accurate assessment: all four variants detected in MAP2K2 fell into the VUS category and approximately half of the variants detected in HRAS $(2 / 3)$, KRAS (1/3), MAK2K1 (2/4), and SHOC2 (2/5) were VUS (Supplementary Table S1).

Among 36 identified VUS, SOS1 had the highest genespecific VUS contribution of $30.6 \%$ (11), followed by PTPN11 and RAF1 at $13.9 \%$ (5) each. BRAF and MAP2K2 each contributed $11.9 \%$ (4), and the rest of the genes were minor contributors: $5.6 \%$ (2) for HRAS, MAP2K1, and SHOC2 each, and $2.8 \%$ (1) for KRAS (Fig. $2 \mathbf{b}$ ).

The overall positive rate or diagnostic yield for 409 postnatal specimens was $21.3 \%$ (87) and the overall VUS yield was $8.8 \%$ (36). In one case two VUS were identified, whereas in six cases with two variants, a pathogenic variant was found in addition to the VUS. Therefore, only $7.3 \%$ (30) of postnatal cases produced an inconclusive test report due to VUS.

\section{Parental studies}

Follow-up studies of both parents were possible for 39 prenatal and 8 postnatal cases. A total of 22 pathogenic and likely 
a

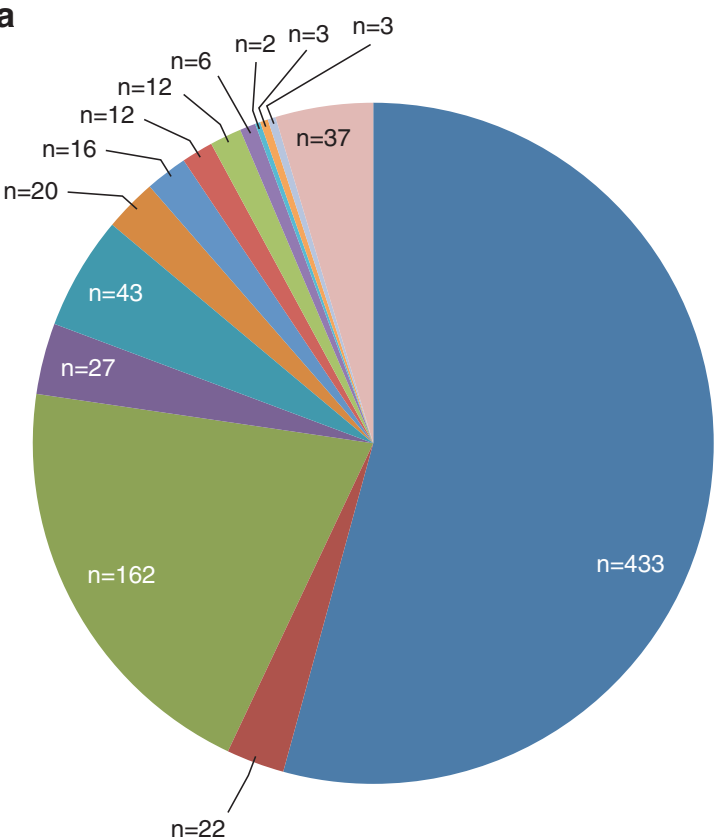

Increased NT alone

Increased NT and other ultrasound findings

Cystic hygroma alone

Cystic hygroma and other ultrasound findings

Thickened nuchal fold alone

Thickened nuchal fold and other ultrasound findings

Heart defect alone

Heart defect and other ultrasound findings (excluding increased NT, cytic hygroma, and thickened nuchal fold)

Fetal hydrops/edema/pleural effusion

Fetal hydrops/edema and other ultrasound findings (excluding increased NT, cystic hygroma, and heart defect)

Polyhydramnios alone

Renal anomalies

Fetal demise only

Other abnormal ultrasound findings b

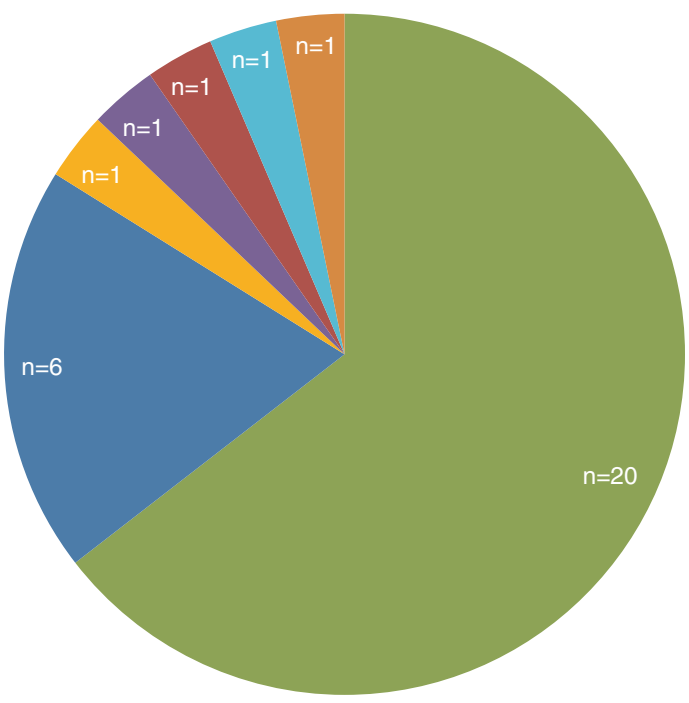

Cystic hygroma alone

Increased NT alone

Bilateral pleural effusion, upper body diffuse skin edema and heart defect

Cystic hygroma and fetal demise

Increased NT and upper body lymphedema

Family history of NSRC

Pleural effusion, ascites and heart defect

Fig. 3 Ultrasound findings in prenatal NSRC studies. a Distribution of ultrasound findings in prenatal specimens. b Ultrasound findings in fetuses with a positive result. NT nuchal translucency, NSRC Noonan syndrome and related conditions

pathogenic variants and 25 VUS were assessed to determine the origin of each variant. The results showed that $90.9 \%$ (20) of the pathogenic and likely pathogenic variants originated de novo, whereas $84 \%$ (21) of the VUS were inherited (Table 1).

\section{Recurrent variants}

Recurrent pathogenic and likely pathogenic variants across both groups were identified, with four recurrent variants in PTPN11 found only among prenatal specimens (Table 2).

\section{DISCUSSION}

Since the identification of PTPN11 in the etiology of Noonan syndrome, molecular genetic testing has been an important adjunct to clinical diagnosis. Molecular testing has evolved into multiple-gene panels as understanding of clinical 
relatedness among NSRCs deepened and more causative genes were identified. In recent years, testing has extended to include prenatal diagnosis, as several ultrasound markers have been shown to be overrepresented in fetuses affected by NSRCs. ${ }^{5,6}$ One such ultrasound marker is increased NT, which is detected during first trimester screening and is the most common fetal ultrasound anomaly triggering further genetic testing. ${ }^{36}$ This places NSRCs testing among the most frequently ordered prenatal tests.

Our main interest was to assess and compare the pattern of gene-specific involvement and the spectrum of identified variants between prenatal and postnatal groups. We analyzed the outcomes of NSRCs molecular testing in 1254 specimens encompassing 845 prenatal and 409 postnatal cases. A ninegene panel including BRAF, HRAS, KRAS, MAP2K1, MAP2K2, PTPN11, RAF1, SOS1, and SHOC2 was used in this study.

Table 1 Parental follow-up studies

\begin{tabular}{llll} 
Variant classification & \multicolumn{2}{l}{ Variant origin } \\
\cline { 2 - 4 } & De novo & Maternal & Paternal \\
\hline Pathogenic & 17 & 2 & 0 \\
Likely pathogenic & 3 & 0 & 0 \\
VUS-possibly pathogenic & 2 & 0 & 1 \\
VUS-uncertain significance & 2 & 8 & 6 \\
VUS-possibly benign & 0 & 4 & 2 \\
\hline
\end{tabular}

VUS variant of uncertain significance
Our data show that the gene-specific pattern of overall variant contribution has similarities between the two groups. In both cohorts, PTPN11 harbored the majority of observed variants, $37.8 \%$ in prenatal and $55.3 \%$ in postnatal, and SOS1 had the second largest number of variants, contributing $27 \%$ of total variants in prenatal and $14.6 \%$ in postnatal cases (Fig. 1). Also in both groups, RAF1 and BRAF were among the next most common variant contributors with approximately 8 and $7 \%$ of total variants, respectively; whereas $H R A S$, KRAS, MAP2K1, and SHOC2 were among the smallest $(<5 \%)$ contributors (Fig. 1). The gene-specific contribution of pathogenic and likely pathogenic variants and VUS also showed similarities: PTPN11 was the highest contributor of pathogenic and likely pathogenic variants $(80.7 \%$ in prenatal and $72.4 \%$ in postnatal specimens), and had the lowest VUS frequency $(10.7 \%$ in prenatal and $7.4 \%$ in postnatal specimens) (Fig. 2, Supplementary Table S1). SOS1 was the highest VUS contributor in both groups: $44.2 \%$ in prenatal and $30.6 \%$ in postnatal specimens (Fig. 2, Supplementary Table S1).

However, the overall VUS frequency was twice as high in prenatal specimens compared with postnatal specimens: 58.1 vs. 29.3\%. PTPN11, a major and well-studied disease contributor with a highly conserved structure and an expected low VUS frequency, nevertheless displayed the same trend in having a higher VUS frequency in the prenatal cohort (10.7 vs. $7.4 \%$ ). SOS1 also showed an over 1.5-fold difference in gene-specific VUS frequency between prenatal and postnatal cohorts (95 vs. $61.1 \%$ ). Thus, the classification spectrum of observed variants appears to be influenced by the prenatal/

Table 2 Recurrent variants

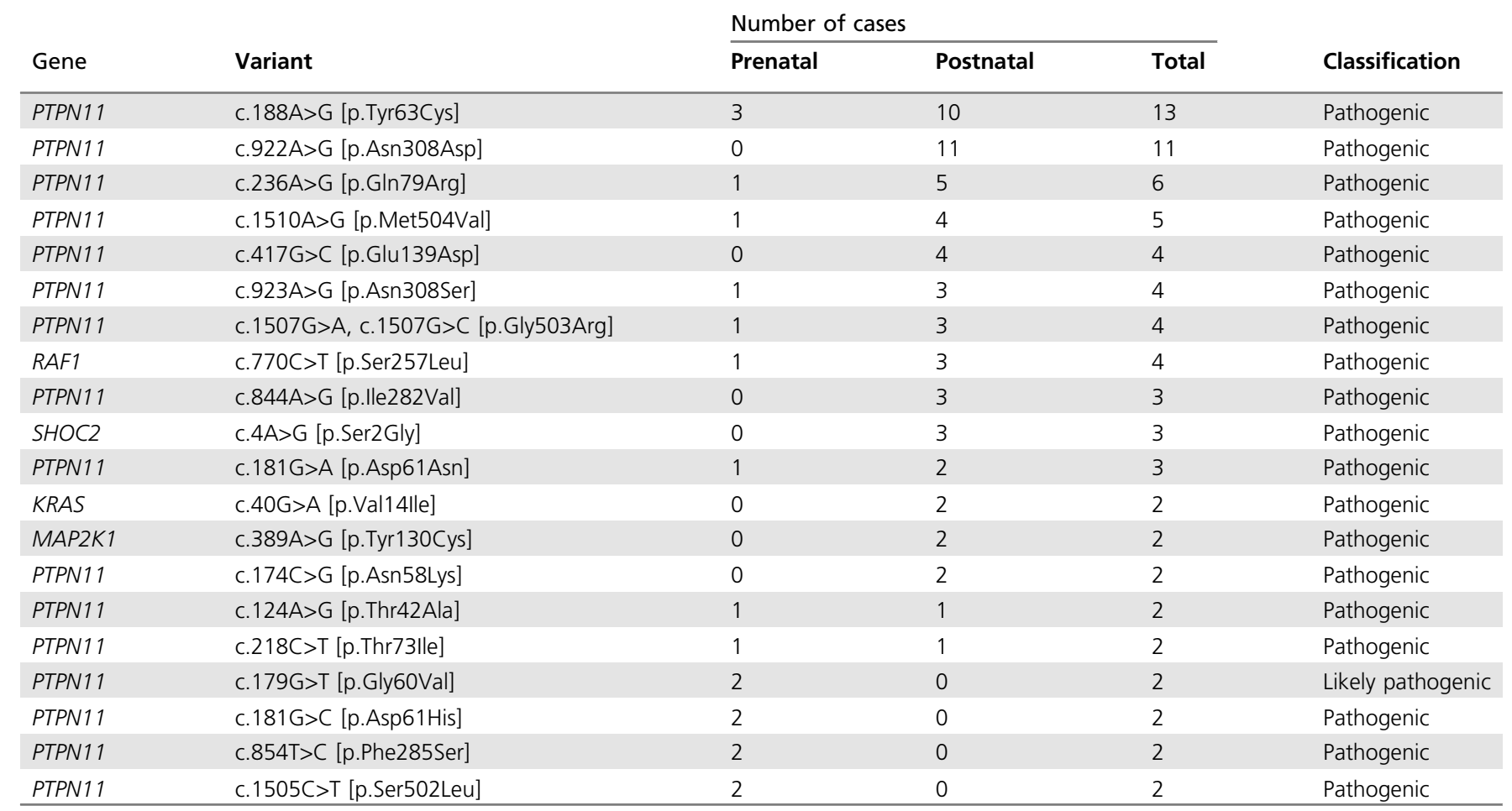


postnatal context of the specimen. Interestingly, the genespecific VUS contribution does not seem to correlate inversely with the degree of the gene's involvement with NSRCs. For example, PTPN11, the most commonly mutated gene in NSRCs, has the lowest VUS frequency, whereas SOS1, the second most commonly mutated gene, has one of the highest gene-specific VUS frequencies. A longitudinal study following up fetuses with identified VUS for postnatal assessment may further the understanding for the difference in VUS frequency between prenatal and postnatal cohorts.

It is important to note that "VUS" is a transient variant classification category that is anticipated to change to a more definitive clinical interpretation as general population sequencing data are further collected and more variant data are submitted to the ClinVar database (https://www.ncbi.nlm.nih. gov/clinvar/). Identification of a VUS, especially in the prenatal setting, creates concern as it is not viewed as a reassuring result. Parental follow-up studies are always recommended to assess the origin of a detected VUS. A de novo occurrence of a VUS strengthens the evidence of pathogenicity, and could change its classification category. Thus, determination of a VUS origin is of importance for its proper classification. It has to be noted that interpretation of a familial VUS is frequently hindered by the lack of clinical assessment of a carrier parent. NSRCs could be underdiagnosed due to variable expressivity and a parental diagnosis could be made after a diagnosis in their offspring. ${ }^{37}$ Therefore, if a familial VUS is identified in a prenatal study, parental assessment by a clinical geneticist is important in delineation of its clinical significance. In our study, $90.9 \%$ of the pathogenic and likely pathogenic variants originated de novo, whereas $84 \%$ of the VUS were inherited (Table 1 ).

Variants were detected in only $8.5 \%$ of prenatal cases in comparison with $28.4 \%$ of postnatal cases. The VUS yield was $5.1 \%$ in prenatal and $8.8 \%$ in postnatal cases. The diagnostic yield differed considerably between the two groups, being $3.7 \%$ in prenatal and $21.3 \%$ in postnatal cases. When prenatal cases were further separated into subgroups with cystic hygroma and increased NT as the sole ultrasound findings, the diagnostic yield became 12.3 and $1.4 \%$, respectively. Of note, the cystic hygroma and increased NT prenatal subgroups also showed differences in their gene-specific distribution of pathogenic and likely pathogenic variants: positive results were observed in PTPN11, RAF1, and SOS1 for the indication of cystic hygroma, and only in PTPN11 and HRAS for the indication of increased NT (Fig. 1b). Most prenatal cases with a positive result had a single abnormal ultrasound finding, predominantly cystic hygroma (20/31), which reinforces the notion that NSRC molecular testing should be considered in fetuses with at least one NSRC related ultrasound finding. ${ }^{38}$ Ultrasound findings observed in the positive prenatal cohort cases are consistent with prior publications, although in our study heart defects were not as predominant $(2 / 31),{ }^{6,38}$ As the exact NT measurements were rarely provided, we could not determine if positive cases had a larger nuchal measurement compared with the other studies. ${ }^{38,39}$ More genes etiological to NSRCs were identified in recent years. ${ }^{26,28-34}$ Although our panel does not include all the currently known NSRC genes, the tested nine genes include the most commonly mutated ones and provide a large data set for a comparative assessment of outcomes between prenatal and postnatal cohorts. A larger panel is expected to improve the overall diagnostic yield, although the relationship between the numbers of tested genes and diagnostic yield should not be expected to be linear because some of the genes have been reported in very few patients.

This study compared the gene-specific pattern and clinical spectrum of variants identified in prenatal and postnatal cohorts referred for molecular NSRCs testing. It provides further data on the expected diagnostic yield for NSRCs, and demonstrates that ascertainment, including the prenatal or postnatal context, and prenatal clinical indication, predicts differences in the variant classification spectrum of identified variants.

\section{ELECTRONIC SUPPLEMENTARY MATERIAL}

The online version of this article (https://doi.org/10.1038/s41436018-0062-0) contains supplementary material, which is available to authorized users.

\section{DISCLOSURE}

Authors are employees of the Integrated Genetics, a specialty genetic testing group of Laboratory Corporation of America Holdings.

\section{REFERENCES}

1. Roberts $A E$, Allanson JE, Tartaglia M, et al. Noonan syndrome. Lancet. 2013;381:333-42

2. Noonan JA. Noonan syndrome and related disorders: alterations in growth and puberty. Rev Endocr Metab Disord. 2006;7:251-5.

3. Jorge AA, Malaquias AC, Arnhold IJ, et al.Noonan syndrome and related disorders: a review of clinical features and mutations in genes of the RAS/ MAPK pathway. Horm Res. 2009;71:185-93.

4. Neumann TE, Allanson J, Kavamura I, et al. Multiple giant cell lesions in patients with Noonan syndrome and cardio-facio-cutaneous syndrome. Eur J Hum Genet. 2009;17:420-5.

5. Lee K, Williams B, Roza K, et al. PTPN11 analysis for the prenatal diagnosis of Noonan syndrome in fetuses with abnormal ultrasound findings. Clin Genet. 2009;75:190-4.

6. Croonen EA, Nillesen WM, Stuurman KE, et al. Prenatal diagnostic testing of the Noonan syndrome genes in fetuses with abnormal ultrasound findings. Eur J Hum Genet. 2013;21:936-42.

7. Tidyman WE, Rauen KA. The RASopathies: developmental syndromes of Ras/MAPK pathway dysregulation. Curr Opin Genet Dev. 2009. 19:230-6.

8. Tartaglia M, Gelb BD, Zenker M. Noonan syndrome and clinically related disorders. Best Pract Res Clin Endocrinol Metab. 2011;25:161-79.

9. Gelb BD, Tartaglia M. Noonan syndrome and related disorders: dysregulated RAS-mitogen activated protein kinase signal transduction. Hum Mol Genet. 2006;15:R220-6.

10. El Bouchikhi I, Belhassan K, Moufid FZ, et al. Noonan syndrome-causing genes: Molecular update and an assessment of the mutation rate. Int J Pediatr Adolesc Med. 2016;3:133-42.

11. Tartaglia M, Mehler EL, Goldberg R, et al. Mutations in PTPN11, encoding the protein tyrosine phosphatase SHP-2, cause Noonan syndrome. Nat Genet. 2001;29:465-8.

12. Lequis $E$, Schrander-Stumpel $C$, Scholleen $E$, et al. PTPN11 mutations in LEOPARD syndrome. J Med Genet. 2002;39:571-4. 
13. Ogata T, Yoshida R. PTPN11 mutations and genotype-phenotype correlations in Noonan and LEOPARD syndromes. Pediatr Endocrinol Rev. 2005;2:669-74.

14. Tartaglia M, Kalidas $K$, Shaw A, et al. PTPN11 mutations in Noonan syndrome: molecular spectrum, genotype-phenotype correlation, and phenotypic heterogeneity. Am J Hum Genet. 2002;70:1555-63.

15. Tartaglia M, Pennacchio LA, Zhao C, et al. Gain-of-function SOS1 mutations cause a distinctive form of Noonan syndrome. Nat Genet. 2007;39:75-79.

16. Denayer E, Devriendt $\mathrm{K}$, de Ravel $\mathrm{T}$, et al. Tumor spectrum in children with Noonan syndrome and SOS1 or RAF1 mutations. Genes Chromosomes Cancer. 2010:49:242-52.

17. Lepri F, De Luca A, Stella L, et al. SOS1 mutations in Noonan syndrome: molecular spectrum, structural insights on pathogenic effects, and genotype-phenotype correlations. Hum Mutat. 2011;32:760-72.

18. Pandit B, Sarkozy A, Pennacchio LA, et al. Gain-of-function RAF1 mutations cause Noonan and LEOPARD syndromes with hypertrophic cardiomyopathy. Nat Genet. 2007;39:1007-12.

19. Sarkozy A, Carta C, Moretti S, et al. Germline BRAF mutations in Noonan, LEOPARD, and cardiofaciocutaneous syndromes: molecular diversity and associated phenotypic spectrum. Hum Mutat. 2009;30: 695-702.

20. Nava C, Hanna N, Michot C, et al. Cardio-facio-cutaneous and Noonan syndromes due to mutations in the RAS/MAPK signalling pathway: genotype-phenotype relationships and overlap with Costello syndrome. $J$ Med Genet. 2007;44:763-71.

21. Aoki $Y$, Niihori $\mathrm{T}$, Kawame $\mathrm{H}$, et al. Germline mutations in HRAS protooncogene cause Costello syndrome. Nat Genet. 2005;37:1038-40.

22. Gripp KW, Lin $A E$, Stabley DL, et al. HRAS mutation analysis in Costello syndrome: genotype and phenotype correlation. Am J Med Genet $A$. 2006;140:1-7

23. Kerr B, Delrue MA, Sigaudy S, et al. Genotype-phenotype correlation in Costello syndrome: HRAS mutation analysis in 43 cases. J Med Genet. 2006:43:401-5

24. Cordeddu V, Di Schiavi E, Pennacchio LA, et al. Mutation of SHOC2 promotes aberrant protein $\mathrm{N}$-myristoylation and causes Noonan-like syndrome with loose anagen hair. Nat Genet. 2009:41:1022-6.

25. Zenker M, Lehmann K, Schulz AL, et al. Expansion of the genotypic and phenotypic spectrum in patients with KRAS germline mutations. J Med Genet. 2007;44:131-5.
26. Yamamoto GL, Aguena $\mathrm{M}$, Gos $\mathrm{M}$, et al. Rare variants in SOS2 and LZTR1 are associated with Noonan syndrome. J Med Genet. 2015;52:413-21.

27. Aoki $Y$, Niihori T, Inoue S, Matsubara $Y$. Recent advances in RASopathies. J Hum Genet. 2016;61:33-39.

28. Aoki Y, Niihori T, Banjo T, et al. Gain-of-function mutations in RIT1 cause Noonan syndrome, a RAS/MAPK pathway syndrome. Am J Hum Genet. 2013;93:173-80.

29. Cirstea IC, Kutsche K, Dvorsky $R$, et al. A restricted spectrum of NRAS mutations causes Noonan syndrome. Nat Genet. 2010;42:27-9.

30. Cordeddu V, Yin JC, Gunnarsson C, et al. Activating mutations affecting the Dbl homology domain of SOS2 cause Noonan syndrome. Hum Mutat. 2015:36:1080-7.

31. Chen PC, Yin J, Yu HW, et al. Next-generation sequencing identifies rare variants associated with Noonan syndrome. Proc Natl Acad Sci USA. 2014b;111:11473-8

32. Vissers LE, Bonetti M, Paardekooper Overman J, et al. Heterozygous germline mutations in $A 2 M L 1$ are associated with a disorder clinically related to Noonan syndrome. Eur J Hum Genet. 2015;23:317-24.

33. Flex $E$, Jaiswal $M$, Pantaleoni $F$, et al. Activating mutations in RRAS underlie a phenotype within the RASopathy spectrum and contribute to leukaemogenesis. Hum Mol Genet. 2014;23:4315-27.

34. Gripp KW, Aldinger KA, Bennett JT, et al. A novel rasopathy caused by recurrent de novo missense mutations in PPP1CB closely resembles Noonan syndrome with loose anagen hair. Am J Med Genet $A$. 2016;2016(170A):2237-47

35. Richards S, Aziz N, Bale $S$, et al. Standards and guidelines for the interpretation of sequence variants: a joint consensus recommendation of the American College of Medical Genetics and Genomics and the Association for Molecular Pathology. Genet Med. 2015;17:405-24.

36. Devine PC, Malone FD. First trimester screening for structural fetal abnormalities: nuchal translucency sonography. Semin Perinatol. 1999:23:382-92.

37. Bertola DR, Pereira AC, de Oliveira PS, et al. Clinical variability in a Noonan syndrome family with a new PTPN11 gene mutation. Am J Med Genet A. 2004;130:378-83.

38. Hakami F, Dillon MW, Lebo $M$, et al. Retrospective study of prenatal ultrasound findings in newborns with a Noonan spectrum disorder. Prenat Diagn. 2016;36:418-23

39. Ali MM, Chasen ST, Norton ME. Testing for Noonan syndrome after increased nuchal translucency. Prenat Diagn. 2017;37:750-3. 